\title{
Isolation and Antibiotic-Resistant Pattern of Opportunistic Infectious Microbes from the Infected Sites of Oral Cancer Patients Compared to That of Healthy People Oral Microbiota
}

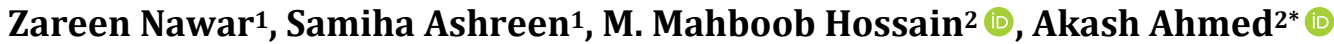 \\ ${ }^{1}$ Biotechnology Program, Department of Mathematics and Natural Sciences, Brac University, Dhaka, Bangladesh \\ ${ }^{2}$ Microbiology Program, Department of Mathematics and Natural Sciences, Brac University, Dhaka, Bangladesh \\ Email: *akash.ahmed@bracu.ac.bd
}

How to cite this paper: Nawar, Z., Ashreen, S., Mahboob Hossain, M. and Ahmed, A. (2021) Isolation and Antibiotic-Resistant Pattern of Opportunistic Infectious Microbes from the Infected Sites of Oral Cancer Patients Compared to That of Healthy People Oral Microbiota. Advances in Microbiology, 11, 343-359.

https://doi.org/10.4236/aim.2021.118027

Received: June 24, 2021

Accepted: July 27, 2021

Published: July 30, 2021

Copyright $\odot 2021$ by author(s) and Scientific Research Publishing Inc. This work is licensed under the Creative Commons Attribution International License (CC BY 4.0).

http://creativecommons.org/licenses/by/4.0/

\begin{abstract}
Introduction: Oral cancer is the third most prominent type of cancer in Bangladesh. During or after oral cancer treatment, immune-compromised cancer patients may be susceptible to an infection by multi-drug-resistant opportunistic microbes. This study aims to identify the prevalent microorganisms from the infected site of oral cancer patients and observe their antibiotic susceptibility pattern. Materials and Methods: Clinical samples were collected from the infected sites of oral cancer patients and healthy people. The swabs collected were placed on nutrient agar slant, then incubated for 24 hours at $37^{\circ} \mathrm{C}$. Bacteria from the slant were inoculated in several selective media (Mannitol Salt Agar Media, KF Streptococcus Agar media, Cetrimide Agar, Eosin Methylene Blue Agar). Several biochemical tests identified opportunistic microorganisms. Finally, the antibiotic susceptibility test was performed by the Kirby Bauer disc diffusion method. Results: The study found that $65.4 \%$ of microbes isolated from the patients' oral cavities were Gram-negative bacteria, and $34.6 \%$ were Gram-positive bacteria. Among the patient group isolates $(\mathrm{n}=55)$, the most prevalent organism was Pseudomonas spp. (30; 54.54\%). Others were Klebsiella spp. (27; 49.09\%), Staphylococcus spp. (24; 43.63\%), E. coli (14; 25.45\%), Streptococcus spp. (14; 25.45\%), Proteus spp. (12; 21.8\%) and Enterococcus spp. (6;10.90\%). Both Gram-positive and Gram-negative microbes from the patient group have shown high resistance to the commonly used antibiotics. In the control group $(n=50)$, the most prevalent organism was Staphylococcus spp. $(15 ; 30 \%)$. Other organisms were Streptococcus spp. (6;12\%), Klebsiella spp. (11;22\%), E. coli (3; 6\%), Streptococcus spp. $(14 ; 25.45 \%)$, Pseudomonas spp. $(8 ; 16 \%)$. The microbes of the
\end{abstract}


control group showed less resistance to the antibiotics and rather showed sensitivity to them. Conclusion: The study revealed a high prevalence of multi-drug-resistant opportunistic microbes on immune-compromised oral cancer patients compared to microbes isolated from healthy people's oral cavity.

\section{Keywords}

Antibiotic Resistance, Gram-Negative Bacteria, Gram-Positive Bacteria, Immuno-Compromised, Oral Cancer

\section{Introduction}

Maintaining good oral health is an integral part of human health. However, at present, oral cancer is one of the most critical health issues. This cancer is the sixth most common cancer worldwide, and every year, half a million people are diagnosed with this type of cancer. It is prevalent in areas where betel quid chewing, smoking, and alcohol drinking are observed in high frequency. Forty percent of cancer cases in Southeast Asia refer to oral cancer [1]. Cancer develops in the anterior tongue, gingival, buccal cavity, retromolar trigone, hard palate, salivary glands, and even tonsil glands [2]. Primarily, oral cancer arises as a lesion that is hyperplastic in growth. Due to external carcinogenic stimuli and the absence of internal cell regulations mechanism because of tumor repressor genes, the hyperplasia turns into metaplasia and anaplasia, leading to malignant invasion. Oral infection caused by the Herpes virus, Human Papillomavirus, Candida albicans, Treponema pallidum, and even poor oral hygiene can also be a factor that increases the risk of oral cancer [3].

In Bangladesh, the rate of oral cancer is high. A study revealed that cancer cases are approximately 200,000 per year and among them, oral cancer represents $20 \%$, and it is the third leading cancer occurring in this country [4]. A study also showed that 7000 people in Bangladesh are diagnosed with oral cavity cancer every year and among them, $6.6 \%$ without feasible cure. The majority of oral cancer patients are from rural areas of Bangladesh [5]. The main causes of oral cancer in Bangladesh are tobacco, betel leaf, catechu, alcohol, and smoking. At the same time, arsenic-contaminated groundwater, availability of chemical carcinogens, mainly formalin-treated fruits, and poor hygiene conditions increase the risks of oral cancer in Bangladesh.

Despite significant development in oral cancer treatment, cancer patients remain at risk of developing severe infections. The immune-compromised patients are susceptible to infections by drug-resistant opportunistic microbes like Staphylococcus aureus, Pseudomonas aeruginosa, Klebsiella, E. coli. This infection may occur during the cancer progressions or after surgery [6]. The infection decreases the recovery rate of patients and it also increases the mortality rate. Due to this infection, cancer also spreads to the other parts of the body. After che- 
motherapy and radiotherapy treatment, the cancer patients become more immune-suppressed and thus reduce the number of white blood cells [7]. The lower level of neutropenia due to this radiotherapy increases the possibility of infectious disease. Significant parameters are involved in oral cancer's etiology, such as age, gender, food habit, race, tobacco use, and alcohol consumption.

For treating infected oral cancer patients, all kinds of antibiotics can be used. Many antibiotics are resistant to both gram-positive and gram-negative bacteria. Sometimes a mixture of antibiotics is used for better treatment. The antibiotics generally used are cephalosporins, aminoglycosides, quinolones, carbapenems, penicillin, and several other antibiotic classes [8]. Antibiotics for which millions of lives have been saved in the past are now less effective against many bacterial infections. The antibiotic resistance crisis is increasing day by day because of the overuse and misuse of these medications. Again, now the microbes are no longer susceptible to the commonly used antibiotics. By the process of mutation, the bacteria can evade the effect of the antibiotics. Through the process of natural selection, those bacteria may carry on and pass the resistant genes into the remaining gene pool [9].

\section{Methodology}

Study populations, place, and duration: The study involved the collection of both data through a questionnaire and a swab sample from the oral cavity. Data and swab samples were collected from 55 oral cancer patients taking treatment from the National Institute of Cancer Research and Hospital, Dhaka, Bangladesh, from August 2019 to February 2020. The clinical samples were checked for the presence of infection on their cancer site. Another control group of 50 people was set with healthy volunteers aged over 22 who did not exhibit any cancer signs (Figure 1).

\section{Antibiotic Susceptibility Test}

According to the guidelines provided by the Clinical Laboratory Standard Institute (CLSI), the antibiotic susceptibility pattern of the isolates was examined using the disc diffusion method [10] [11]. The isolates' 22 hours new culture (Pseudomonas spp., Klebsiella spp., Escherichia coli and Proteus spp., Staphylococcus spp., Streptococcus spp., Enterococcus spp.) were adjusted to the turbidity of $1 \mathrm{McF}$ arland standards and bacterial suspensions were spread over Mueller-Hinton agar (MHA). The antibiotic disc used in the study for gram-positive bacteria included amikacin, gentamicin, Imipenem, ceftazidime, amoxicillin, erythromycin, chloramphenicol, linezolid, ciprofloxacin, nalidixic acid, oxacillin, cloxacillin, and metronidazole (oxoid). Moreover, for gram-negative bacteria, antibiotics involved were amikacin, gentamicin, Imipenem, Ceftriaxone, vancomycin, azithromycin, amoxicillin, penicillin-G, amoxiclav, ciprofloxacin, nalidixic acid, tetracycline, and metronidazole (oxoid). The plates were incubated at $37^{\circ} \mathrm{C}$ for 18 - 20 hours and the zone of inhibitions was measured ( $\mathrm{mm}$ ). 


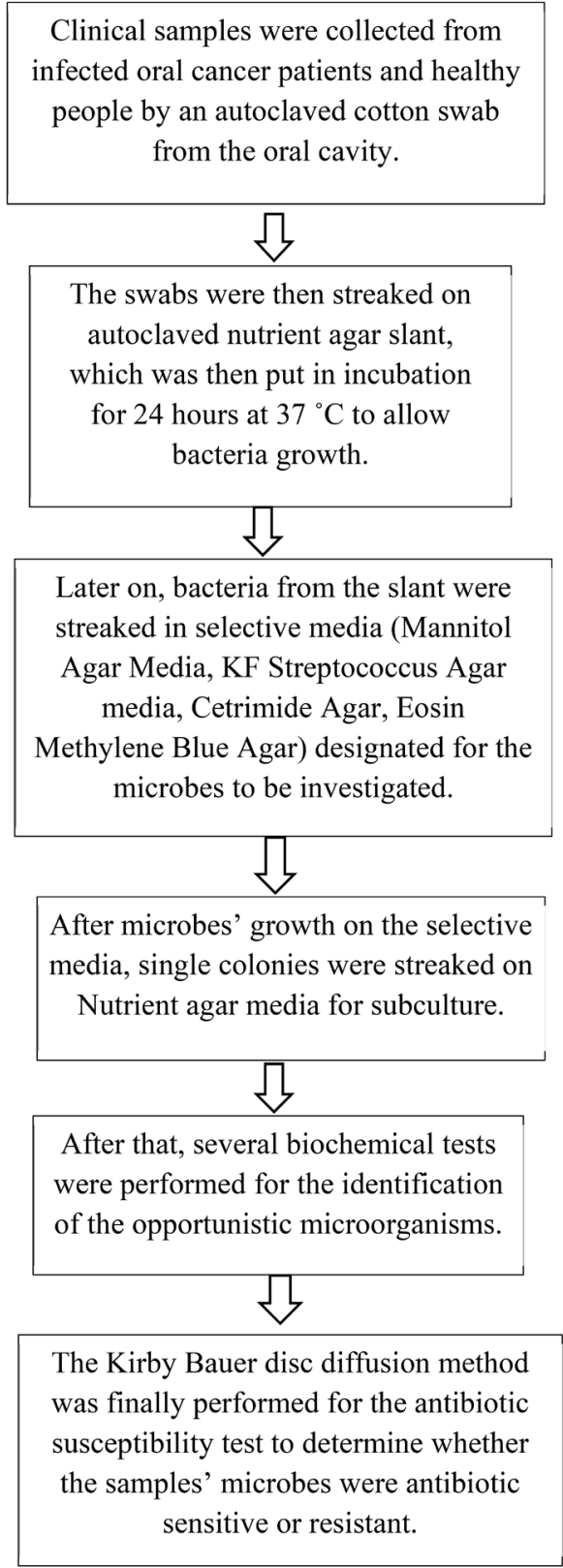

Figure 1. Workflow.

\section{Results}

Result from the growth of selective media: Of the 55 oral cancer patients involved in the study, all of them had an infection in their cancer site. Among them, 24 patients were postoperative patients and the remaining 31 were pre-operative patients. All the specimens from both pre-operative and post-operative patients exhibited bacterial growth on at least one selective media. Among those bacterial growths, $(83 ; 65.4 \%)$ isolates were gram-negative bacteria and $(44 ; 34.6 \%)$ isolates were Gram-positive bacteria. Among the Gram-positive bacteria, the most predominant bacteria were Staphylococcus spp. (24; 43.63\%), followed by Streptococcus spp. $(14 ; 25.45 \%)$ and Enterococcus spp. $(6 ; 10.90 \%)$. Staphylo- 
coccus spp. is the highest found Gram-positive bacteria and in pre-operative patients, the number of Staphyloccocus spp. was more. Moreover, the most prevalent Gram-negative bacteria were Pseudomonas spp. (30; 54.54\%) followed by Klebsiella spp. (27; 49.09\%), E. coli (14; 25.45\%), and Proteus spp. (12; 21.81\%). Most isolated Gram-negative bacteria is Pseudomonas spp. and it is more prevalent in pre-operative patients. Again, of the 50 samples collected from the healthy people, which were considered the control group, 30 showed growth on the selective media used to isolate selected opportunistic pathogens. Among the pathogens found, 21 isolates were Gram-positive bacteria and 22 isolates were Gram-negative bacteria (Table 1).

Identification of isolates from biochemical tests results. The individual colonies found from the selective media were streaked on nutrient agar to observe the colony morphology. Of the 127 isolated bacterial colonies from cancer patients, 83 isolates were Gram-negative bacteria and 44 isolates were Gram-positive bacteria. Again, 43 isolates were retrieved from 30 samples of the control group; 21 isolates were Gram-positive bacteria and 22 isolates were Gram-negative bacteria. Later, microorganisms were identified by standard biochemical tests (Table 2).

Antibiotic susceptibility pattern of the isolates. The antibiotics used in the hospital for controlling the infection were selected for the susceptibility testing. The study tried to unveil the efficacy of the 13 commonly available antibiotics from 11 different groups for both gram-positive and gram-negative bacteria.

All the Gram-negative isolates from cancer patients were 100\% resistant to Vancomycin, Amoxicillin, Penicillin G, and Metronidazole. The isolates' resistance was followed by Azithromycin with $92.9 \%$ resistance, Nalidixic acid with 89.3\% resistance, Tetracycline $88.1 \%$ resistance, and Amoxiclav $81 \%$ resistance. Isolates showed $16.7 \%$ resistance to Imipenem, $7.1 \%$ to Amikacin, and minimum resistance of $2.4 \%$ to Gentamicin (Figure 2).

The overall resistance shown isolated Klebsiella spp. from oral cancer patients was the maximum (70.9\%) and Pseudomonas spp. has shown the least resistance (63.8\%) among all the isolated Gram-Negative bacteria (Table 3).

Again, all the Gram-positive isolates from cancer patients were resistant to Metronidazole, Erythromycin, Amoxicillin, Cloxacillin, Oxacillin. The isolates'

Table 1. Growth of isolates in selective media.

\begin{tabular}{|c|c|c|c|c|c|}
\hline Type of bacteria & Post-op & Pre-op & $\begin{array}{l}\text { Control } \\
\text { group }\end{array}$ & Organism & Media \\
\hline \multirow{3}{*}{$\begin{array}{c}\text { Gram-positive } \\
\text { bacteria }\end{array}$} & 11 & 13 & 15 & Staphylococcus spp. & MSA media \\
\hline & 5 & 9 & 6 & Streptococcus spp. & KF Streptococcus Agar media \\
\hline & 2 & 4 & - & Enterococcus spp. & KF Streptococcus Agar media \\
\hline \multirow{4}{*}{$\begin{array}{c}\text { Gram-negative } \\
\text { bacteria }\end{array}$} & 10 & 17 & 11 & Klebsiella spp. & EMB media \\
\hline & 6 & 6 & - & Proteus spp. & EMB media \\
\hline & 5 & 9 & 3 & E. coli & EMB media \\
\hline & 12 & 18 & 8 & Pseudomonas spp. & Cetrimide media \\
\hline
\end{tabular}




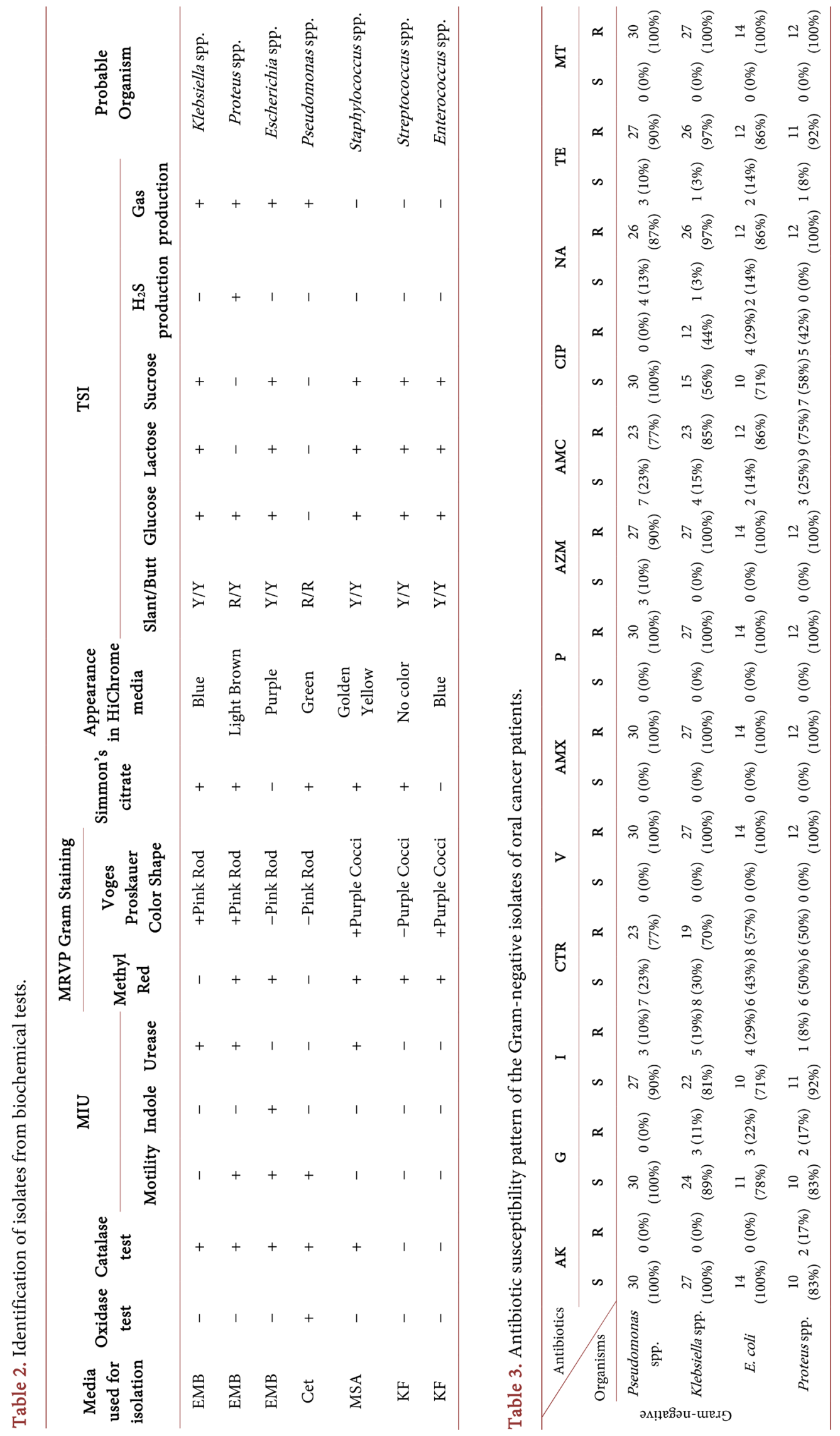


resistance was followed by Nalidixic acid with a percentage of $95.5 \%$ resistance and Ceftazidime with $90.9 \%$ resistance. In contrast, the Gram-positive bacteria isolated from cancer patients showed $13.6 \%$ resistance to Amikacin and Gentamicin, 3.8\% resistance to Ciprofloxacin and 34\% resistance to Linezolid. The isolated Gram-positive isolates from oral cancer patients showed no resistance against Imipenem (Figure 3).

The overall resistance shown by the isolated Enterococcus spp. from oral cancer patients was the maximum (61.5\%) and Streptococcus spp. has shown the least resistance (59.3\%) among all the isolated Gram-positive bacteria (Table 4).

The control group had 50 specimen samples, from which 30 samples have shown growth. Among the 43 isolates 21 isolates were Gram-positive bacteria and 22 isolates were Gram-negative bacteria.

Here, all of the 22 Gram-negative isolates showed $100 \%$ resistance to Vancomycin, Metronidazole, Amoxicillin, Penicillin. No resistance was found in the

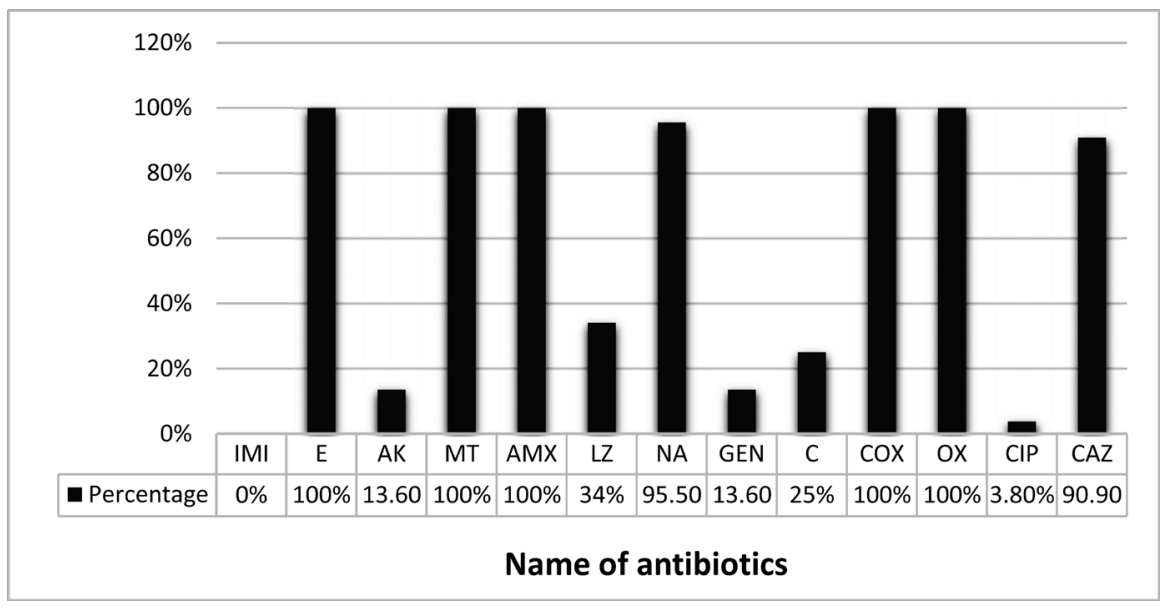

Figure 2. Antimicrobial resistance pattern of Gram-negative bacteria isolated from cancer patients.

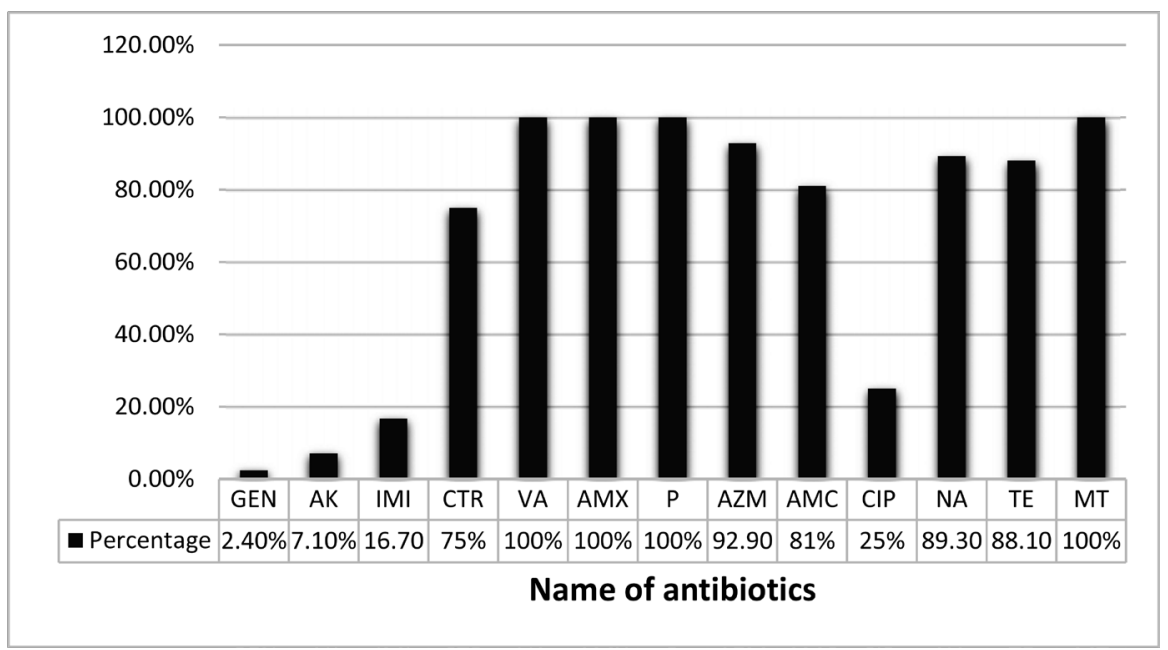

Figure 3. Antimicrobial resistance pattern of Gram-positive bacteria isolated from cancer patients. 


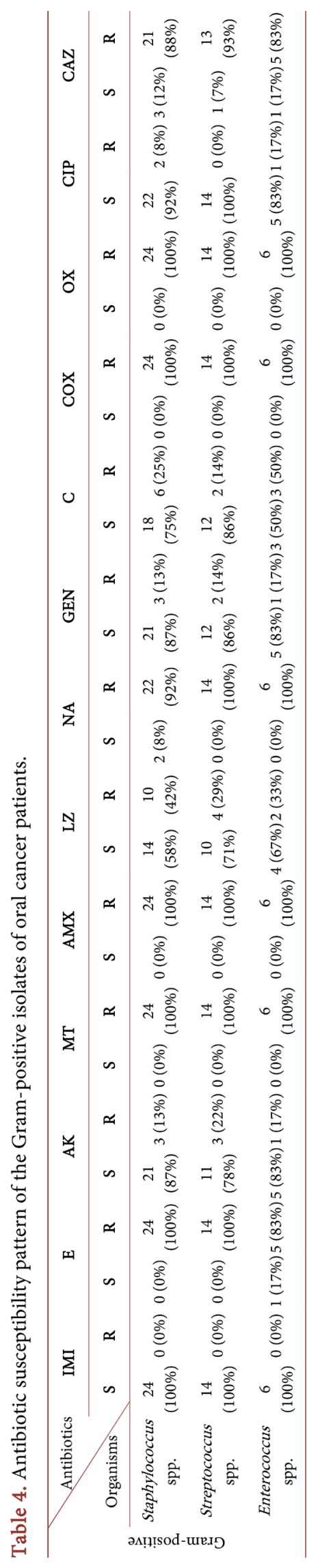


case of Amikacin, Gentamicin, and Ciprofloxacin antibiotic (Figure 4).

The 21 isolated Gram-positive isolates from healthy people showed $100 \%$ resistance to Metronidazole, Amoxicillin, Cloxacillin and Oxacillin bacteria. It showed a low percentage of resistance for Chloramphenicol (38.9\%), Ceftazidime (44.4\%), and Erythromycin (44.4\%). Again, these isolated Gram-positive bacteria were $100 \%$ susceptible that is $0 \%$ resistant to Imipenem, Amikacin, Gentamicin, Ciprofloxacin, and Linezolid (Figure 5).

Analysis of survey according to the questionnaire: In the study majority of the patients involved were male with a percentage of 56.4, while the percentage of female patients was 43.6 (Figure 6).

Again, it is retrieved that oral cancer is more prevalent in the age group of 50 59 (Figure 7).

Lastly, the distribution of predisposing factors in oral cancer patients has shown that almost $52.7 \%$ of patients habitually consume tobacco (Figure 8 ).

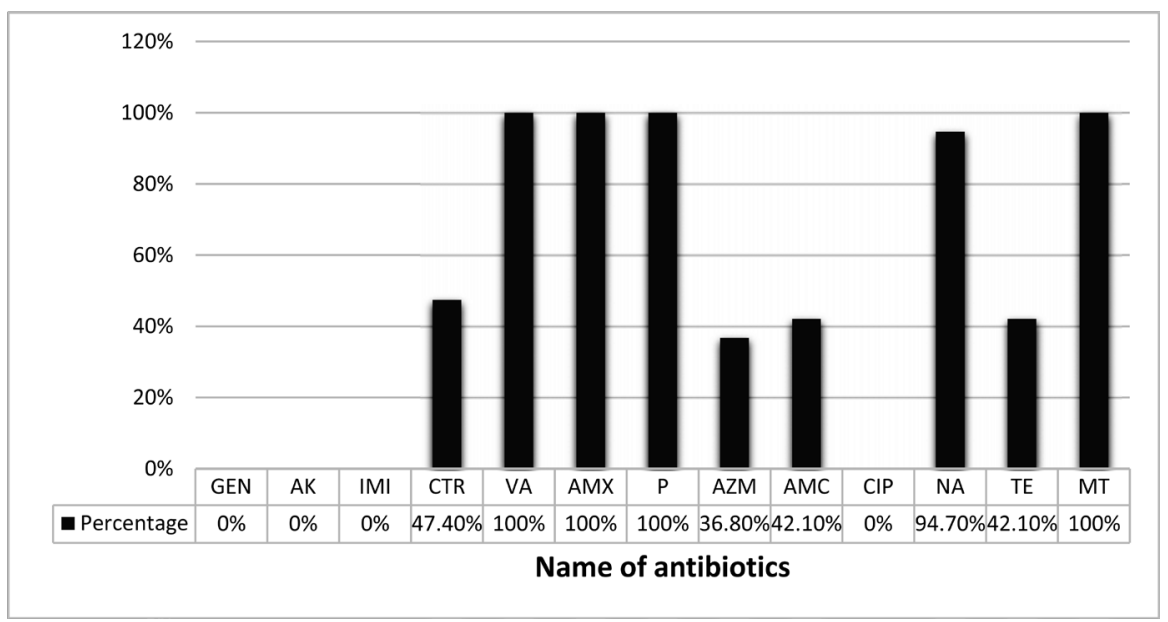

Figure 4. Antimicrobial resistance pattern of Gram-negative bacterial isolates (control group).

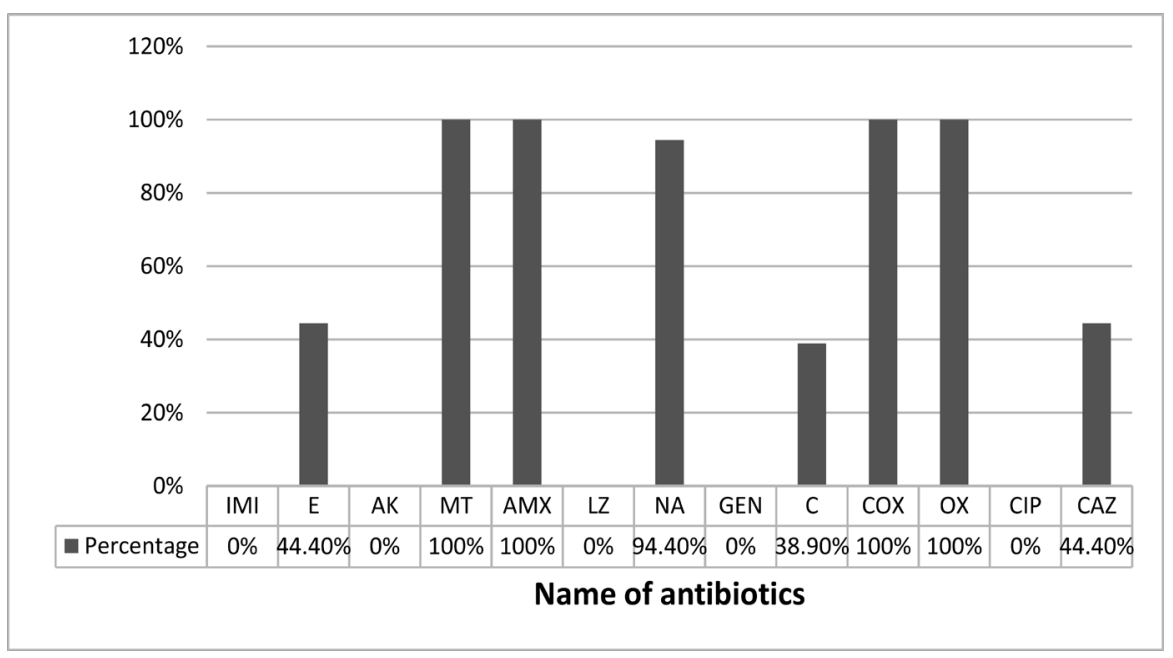

Figure 5. Antimicrobial resistance pattern of Gram-positive bacterial isolates (control group). 


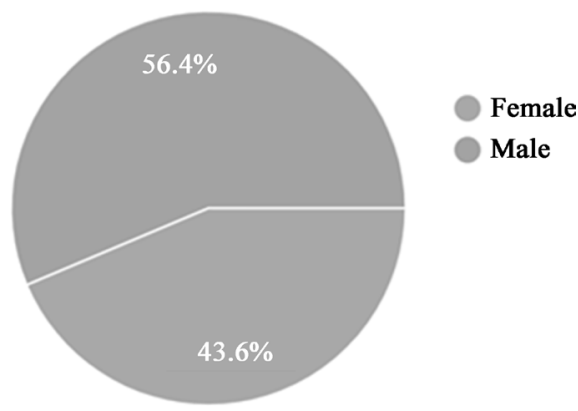

Figure 6. Gender distribution.

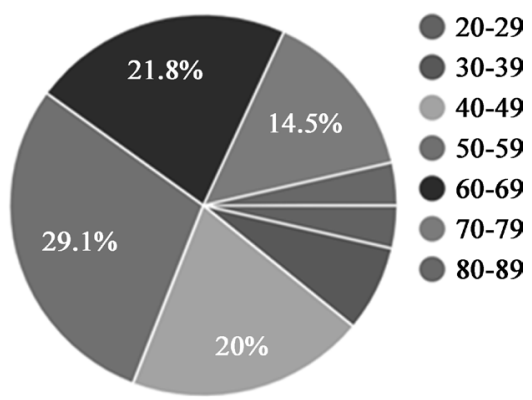

Figure 7. Age group distribution of cancer patients.

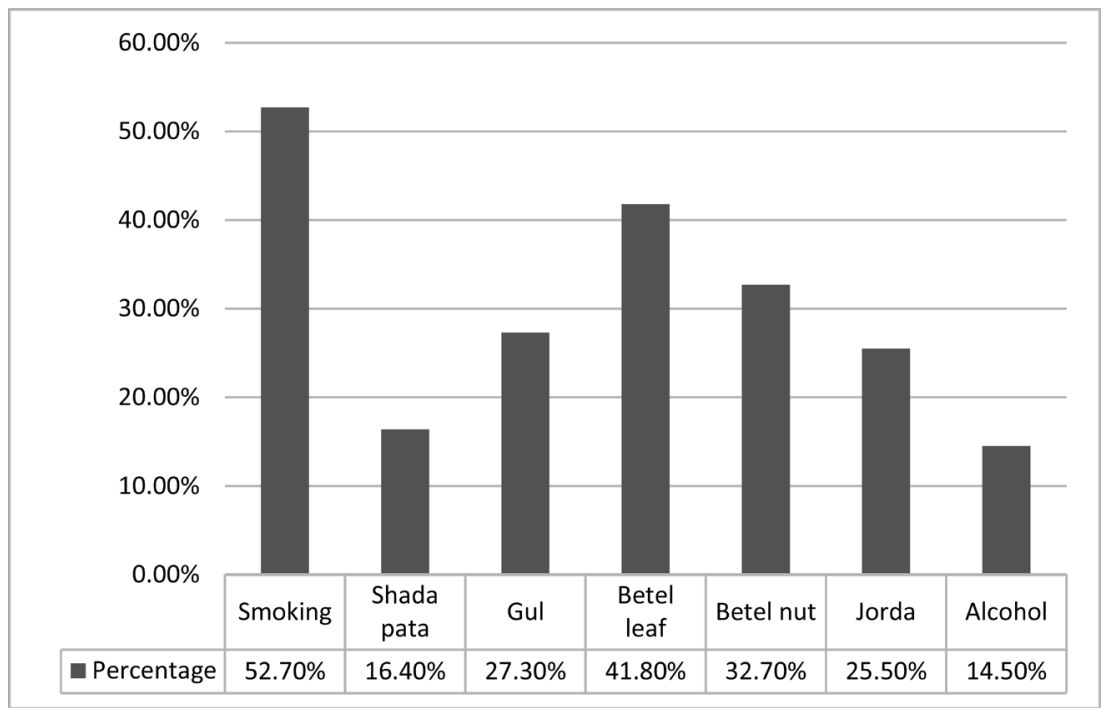

Figure 8. Possible predisposing factors of oral cancer.

The second was the consumption of betel leaf and betel nut. But, in the healthy people, the scenario was different (Figure 9). Healthy people did not have the habit of consuming tobacco like the oral cancer patients.

\section{Discussion}

In the oral cavity is located a blend of microbial species with their own dietary and physicochemical necessities. Bacteria are the most predominant microorganisms present, though fungi, viruses, and protozoa are also found. The salivation in the oral cavity and spit may contain around 100 million of these microscopic 


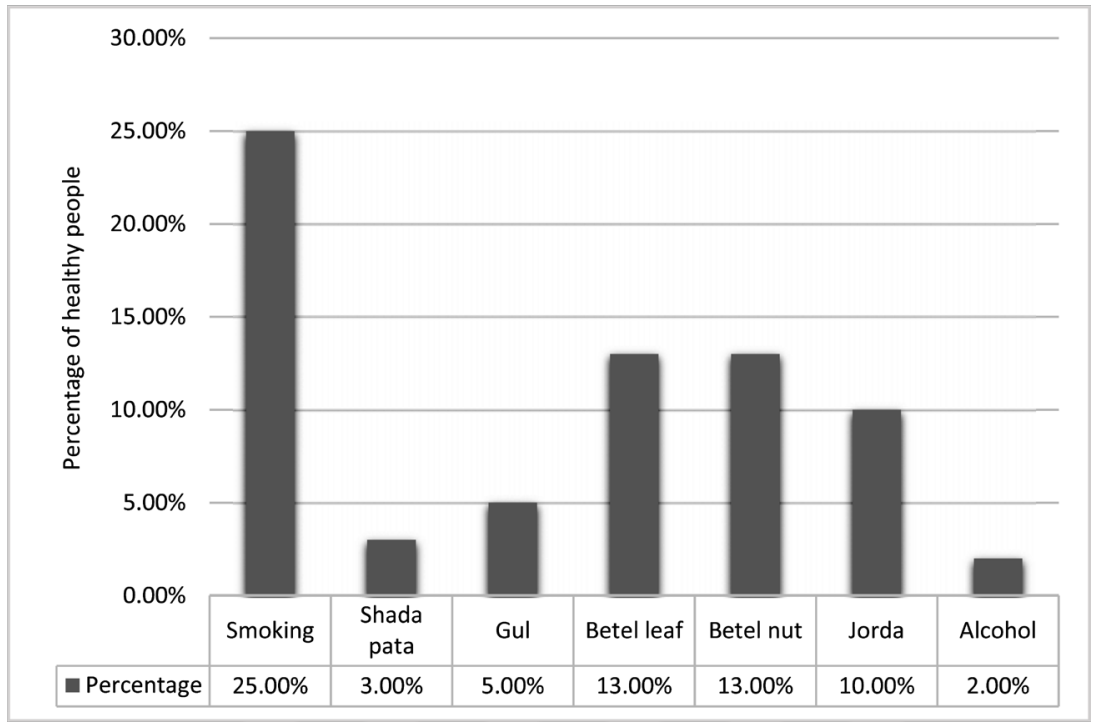

Figure 9. Possible habitual factors of healthy people.

organisms for every milliliter [12]. A microbiological study was performed to identify and isolate opportunistic bacteria from the buccal cavity of oral cancer-infected patients and healthy adults. Oral cancer patients usually develop ulcers in their oral cavity. Opportunistic bacteria develop and infect that ulcer area. For this, the healing process of these kinds of patients becomes long and complicated. The study clearly showed the presence of opportunistic bacteria in the oral cavity of both oral cancer-infected patients and healthy adults and resistance patterns of different kinds of antibiotics.

This study has aimed to develop a protocol for assessing risk factors related to oral cancer infection. The oral cavity is interlinked with the respiratory tract and digestive tract, so organisms that reside there can infect and colonize in the mouth. In contrast, several other studies have reported qualitative changes in oral flora during chemotherapy. This change is also a risk factor for immunocompromised patients [13]. As a result, it can lead to pneumonia, bacteremia, and other health hazards.

It is imperative to have information on the pathogens that dwell in the oral cavity to anticipate dental maladies and the related systematic complications caused by them [14]. Oral cancer patients undergoing chemotherapy and radiotherapy lack immunity. Drug-resistant opportunistic infections cause health problems in this immunocompromised host [15]. Thus, it creates various complexities in oral cancer patients. Gram-positive and Gram-negative bacteria pose a hazard to these weak immune system patients. In a previous study, (61; 63.54\%) Gram-positive bacteria and (41; 42.7\%) Gram-negative bacteria were isolated, with $(28 ; 29 \%)$ Streptococcus spp. is the most prevalent [16]. However, in the present study, $(83 ; 65.4 \%)$ Gram-negative bacteria and (44; 34.6\%) Gram-positive bacteria were isolated, with $(30 ; 54.54 \%)$ Pseudomonas spp. is the most prevalent one.

The statistical analysis has represented that males within the age of $50-59$ 
were more affected by oral cancer than females. Poor oral hygiene, alcohol and tobacco use increase the risk of oral cancers [17]. Tobacco and alcohol consumption are considered the primary risk factor for oral cancer [18]. In the present study, a higher percentage of smokers in oral cancer patients has proved this theory. After smoking, betel leaf and betel nut chewing also significantly impact oral cancer's etiology. This scenario was entirely different for healthy people. Survey has shown that healthy people did not habitually use tobacco, betel leaf, and betel nut.

In the present study the most prevalent Gram-positive bacteria is Staphylococcus spp. Whereas, in a study conducted earlier, only (5; 5.2\%) Staphyloccocus spp. were prevalent. In that study, (28; 29.2\%) Streptococcus spp. were isolated, which was the most prevalent one [16]. Again, in two recent studies, it was illustrated that the most isolated Gram-negative bacteria were Klebsiella spp. (13; $13.5 \%)$ [16] and (37; 45\%) respectively [19]. So indeed, with the change of time and place, the type of bacteria causing infection has changed. In the present study, the percentage of Pseudomonas spp. was higher in pre-operative patients than in post-operative patients. $P$. aeruginosa is responsible for many nosocomial infections and a significant cause of pneumonia [20]. However, in this regard isolates from pre-operative patients, the percentage of Pseudomonas was higher. In several conducted studies, $3 \%-7 \%$ of nosocomial infections were because of this species [21]. Among immunosuppressed patients, such as cancer patients, the pneumonia infection rate increased [22]. However, to comment on whether this high level of prevalence is due to oral cancer or just the hospital, more studies have to be conducted with patients who received surgical treatment.

Again, in the present study, it was also observed that the maximum number of isolates were found in pre-operative $(31 ; 56.4 \%)$ patients than in post-operative (24; $43.6 \%)$ patients. Thus, it proves that most of the patients in our country do not maintain proper oral hygiene. That is why bacteria can develop in their ulcer site even before going into any operation. Due to significant oral environment changes, the balance of oral microbes gets disturbed, leading to infection.

Many diseases are caused due to inadequate water, sanitation, and hygiene facilities and practices, particularly in low-income countries [23]. A study retrieved that poor dental health facilitates the conversion of ethanol to mutagenic acetaldehyde through the metabolic activity of bacterial enzymes, which, in turn, is linked to oral cancer [24]. A significant amount of infection was also found in post-operative patients. Thus, it indicates a heavy presence of bacteria in the hospital's vicinity. Infection occurs in the wounds of oral regions, oropharynx, nasal cavity, and paranasal sinuses areas when it is exposed to microorganisms during or after any operation. A study showed that infection was more profound in post-operative than in pre-operative patients [19]. Infections found in oral cancer patients after surgical excision of the tumor as evidence shows that hospital-associated infections are often spread by the hands of health care workers or contaminated medical devices [25]. However, infection is also caused even 
before surgery which is mainly due to the lower standard of living and lack of proper oral hygiene.

The microbiome in the oral cavity of cancerous patients appears to differ from healthy people. In comparison, 50 swab samples were collected from the control group from where 38 bacteria could be isolated. Over there, the highest percentage was observed in Staphylococcus spp. (15; 34\%), the second-highest was Klebsiella spp. (17; 29\%) followed by Pseudomonas spp. (8; 15\%), Streptococcus spp. $(6 ; 13 \%)$, and $E$. coli $(3 ; 7.8 \%)$. These microbes in the control group can be due to an infection in their oral cavity or poor oral hygiene. Dental plaques can act as a reservoir of many Gram-negative bacilli [26]. The bacterial isolates obtained from the control group could be a source of dental plaque [27]. Although, only cheek swabs were collected and any deep swab was avoided to prevent contamination by the throat microbes. Nevertheless, the major difference in percentage between the prevalence of bacteria in cancer patients and the bacteria in the control group shows the role of immune-suppression as a catalyst in allowing pathogenic microbes to grow [15].

The present study also focused on the antibiotic susceptibility pattern of the opportunistic microbes. The antibiotic susceptibility test was implemented by taking 13 different antibiotics of 11 different groups selected based on Gram-negative and Gram-positive bacteria. After the antibiotic susceptibility test, it was observed that all the Gram-positive bacteria showed $100 \%$ resistance to the antibiotics of Penicillin groups such as Amoxicillin, Cloxacillin and Oxacillin, Metronidazole, and Macrolide group, which includes Erythromycin. A study found that Staphylococcus spp. showed $69.2 \%$ resistance to antibiotic Oxacillin [15]. Thus, now the resistance has increased a lot. The Gram-positive microbes of the present study showed $95.5 \%$ resistance to the antibiotic of the Quinolones group. Minimum resistance was observed for Amikacin, Gentamicin antibiotics, and no resistance for Imipenem antibiotics. S. aureus showed $100 \%$ susceptibility to Amikacin in a previous study [16]. In the present study, it has shown $86.4 \%$ susceptibility, which suggests that this species' resistance against the most potent antibiotics is also increasing. The study also retrieved that, the isolated Enterococcus spp. from oral cancer patients has shown the maximum resistance (61.5\%) and Streptococcus spp. has shown the least resistance (59.3\%) among all the isolated Gram-positive bacteria.

The majority of the microbes that could be isolated were Gram-negative bacteria. The Gram-negative bacteria showed 100\% resistance to Penicillin groups' antibiotics, which included Penicillin-G, Amoxicillin. Similar results were observed for the antibiotics from Glycopeptide (Vancomycin) group and Metronidazole. Most Gram-negative bacteria are intrinsically resistant to Vancomycin because of their outer membranes and different cell wall structures, impermeable for large glycopeptides molecules. Virtually all the anaerobic Gram-negative rods are known to be susceptible to Metronidazole [28]. This emerging resistance of Gram-negative bacteria against Metronidazole can be defined by the 
occurrence of specific resistance genes that code for an alternative set of enzymes that can convert activated forms of Metronidazole into non-toxic derivatives [29]. Gram-negative microbes' resistance was followed in Azithromycin with 92.9\% resistance, Nalidixic acid with $89.3 \%$ resistance, Tetracycline with $88.1 \%$ resistance and Amoxiclav with $81 \%$ resistance. It showed the minimum resistance to amikacin, gentamicin, and Imipenem. A previous study has shown $100 \%$ susceptibility to Carbapenem group antibiotics, including Imipenem [16]. Nevertheless, the present study has shown that the Gram-negative bacteria gave a percentage of 83.4 of susceptibility towards this antibiotic Imipenem. The increase in carbapenem-resistant Gram-negative bacteria worldwide is a matter of great concern [30]. From the study, it is also found that, the isolated Klebsiella spp. from oral cancer patients has shown the maximum resistance $(70.9 \%)$ and Pseudomonas spp. has shown the least resistance (63.8\%) among all the isolated Gram-negative bacteria.

In this study, both the Gram-positive and gram-negative isolates showed maximum resistance to antibiotic Metronidazole, Amoxicillin, Nalidixic acid. It has shown the minimum resistance to antibiotics Amikacin, Gentamicin, Imipenem, and Ciprofloxacin. Previously a study showed that all the bacteria had shown $80 \%$ sensitivity to the antibiotics used [16]. However, in this study, the rate of sensitivity is insufficient. Instead, the microbes have shown a considerable percentage of resistance towards the antibiotics used. Thus, the rise of antibiotic resistance is already evident worldwide [31]. The cause can be explained by the fact that antibiotic resistance varies with population studied and geographic and lifestyle changes.

Antibiotics are used for the treatment and prevention of bacterial infections. The resistance occurs when bacteria change in response to the use of antibiotics used. The rapid emergence of resistant bacteria occurs worldwide, endangering antibiotics' efficacy, which has transformed medicine and saved millions of lives. Antibiotic resistance is now one of the biggest threats worldwide. At present, new resistance mechanisms are emerging and spreading globally, threatening our ability to treat common infectious diseases. Nowadays, bacteria are becoming more drug-resistant due to exposure to these various antibiotics. Moreover, the hospital bacteria especially the multidrug bacteria infect the patient more. Cancer patients also go through many chemotherapies, radiotherapy which can be a reason for being drug-resistant to bacteria. Many oral cancer patients do not complete the antibiotic courses, and some do not maintain proper hygiene. The incident of penicillin resistance is not a new case. The resistance of these antibiotics is reported since 1950 .

Regarding antibiotic resistance, combination therapy of antibiotics can be a suitable alternative to treat opportunistic Gram-negative bacteria. Moreover, the genes responsible for the resistance should be investigated via molecular techniques. Most importantly, that would allow faster detection of antibiotic resistance. Hence, it will provide a quicker administration of the most suitable drug. 
In the present study, it was also observed that the microbes isolated from healthy people were more susceptible to the antibiotics used. The Gram-positive isolates of patients group showed high resistance to the maximum antibiotics used. This differed a lot from the resistance percentage of isolates from healthy people. Again, the same case was observed from the Gram-negative bacteria from patient and control group. The percentage of resistance from patient group is much higher than the percentage of resistance from Gram-negative bacteria isolated from healthy people (control group). Such variation in resistance of isolated microbes between the patient and control groups might be because of the difference in their genes. However, from this, it can be interpreted that the microbes of healthy people are less harmful than the microbes of oral cancer patients.

A variation between the isolates from healthy people and oral cancer patients was also observed when isolating opportunistic bacteria. Out of 50 samples collected from healthy people, only 30 samples formed bacterial colonies, of which only 38 isolates were collected. But out of 55 samples from the cancer patients, all the samples formed bacterial colonies and from which 127 isolates were collected. From such difference, it can be interpreted that because of having a high immunity response and maintaining proper oral hygiene, the growth of opportunistic bacteria in healthy people's oral cavity is less than those of oral cancer patients.

\section{Conclusion}

This study revealed risk factors and life-threatening effects of opportunistic microbes such as Staphylococcus spp., Streptococcus spp., and Enterococcus spp., Pseudomonas spp., Proteus spp., Klebsiella spp., and E. coli. A significant difference was found in the study between the numbers of isolates collected from different sample groups. The successful management of bacteria in infection is of great importance. However, it is still a complex issue. Therefore, the study evaluates the current situation of commonly used antibiotics. Knowing the prevalent type of microorganisms present in infected wounds and their resistance pattern is pertinent to choosing adequate treatment. The data presented here, together with the discussion carried out, can help improve oral cancer infection management.

\section{Acknowledgements}

This work is part of a project funded by the Biotechnology Program, Department of Mathematics and Sciences, BRAC University, Dhaka, Bangladesh.

\section{Conflicts of Interest}

The authors declare no conflicts of interest regarding the publication of this paper.

\section{References}

[1] Rodrigues, V.C., Moss, S.M. and Tuomainen, H. (1998) Oral Cancer in the UK: To 
Screen or Not to Screen. Oral oncology, 34, 454-465. https://doi.org/10.1016/S1368-8375(98)00052-9

[2] Ahmed, F. and Islam, K.M. (1990) Site Predilection of Oral Cancer and Its Correlation with Chewing and Smoking Habit-A Study of 103 Cases. Bangladesh Medical Research Council Bulletin, 16, 17-25. https://doi.org/10.1177\%2F003591576906200637

[3] Cawson, R.A. (1969) Leukoplakia and Oral Cancer. Journal of the Royal Society of Medicine, 62, 610-615. https://doi.org/10.1177\%2F003591576906200637

[4] Shaheed, S.M.I. and Molla, M.R. (1996) Oral Cancer in Bangladesh: Its Aetiology and Histological Grading. Journal of Oral Health, 2, 8-11.

[5] Hussain, S.M.A. (2013) Comprehensive Update on Cancer Scenario of Bangladesh. South Asian Journal of Cancer, 2, 279-284. https://doi.org/10.4103/2278-330X.119901

[6] Cloke, D.J., Green, J.E., Khan, A.L., Hodgkinson, P.D. and McLean, N.R (2004) Factors Influencing the Development of Wound Infection Following Free-Flap Reconstruction for Intra-Oral Cancer. British Journal of Plastic Surgery, 57, 556-560. https://doi.org/10.1016/j.bjps.2004.04.006

[7] Gabrilove, J.L., Jakubowski, A., Scher, H., Sternberg, C., Wong, G., Grous, J., et al. (1988) Effect of Granulocyte Colony-Stimulating Factor on Neutropenia and Associated Morbidity Due to Chemotherapy for Transitional-Cell Carcinoma of the Urothelium. New England Journal of Medicine, 318, 1414-1422. https://doi.org/10.1056/NEJM198806023182202

[8] Ubeda, C. and Pamer, E.G. (2012) Antibiotics, Microbiota, and Immune Defense. Trends in Immunology, 33, 459-466. https://doi.org/10.1016/j.it.2012.05.003

[9] Davison, J. (1999) Genetic Exchange between Bacteria in the Environment. Plasmid, 42, 73-91. https://doi.org/10.1006/plas.1999.1421

[10] Clinical \& Laboratory Standards Institute (2009) Performance Standards for Antimicrobial Susceptibility Testing; 19th Informational Supplement, CLSI M100-S19, Clinical and Laboratory Standards Institute, Wayne.

[11] Ferraro, M.J., Craig, W.A. and Dudley, M.N. (2001) Performance Standards for Antimicrobial Susceptibility Testing. Clinical \& Laboratory Standards Institute, Wayne.

[12] Wade, W.G. (2013) Characterisation of the Human oral Microbiome. Journal of Oral Biosciences, 55, 143-148. https://doi.org/10.1016/j.job.2013.06.001

[13] Whitmore, S.E. and Lamont, R.J. (2014) Oral Bacteria and Cancer. PLoS Pathogens, 10, e1003933. https://doi.org/10.1371/journal.ppat.1003933

[14] Philip, K., Teoh, W.Y., Muniandy, S. and Yaakob, H. (2009) Pathogenic Bacteria Predominate in the Oral Cavity of Malaysian Subjects. Journal of Biological Sciences, 9, 438-444. https://dx.doi.org/10.3923/jbs.2009.438.444

[15] Yamashita, K., Ohara, M., Kojima, T., Nishimura, R., Ogawa, T., Hino, T., et al. (2013) Prevalence of Drug-Resistant Opportunistic Microorganisms in Oral Cavity after Treatment for Oral Cancer. Journal of Oral Science, 55, 145-155. https://doi.org/10.2334/josnusd.55.145

[16] Anjali, K., ArunAnatapadma, B., BastitianThattil, S., Parthiban, R. and Selvamani, M. (2020) Study on Oral Microbial Flora and Antibiotic Sensitivity Pattern among Oral Cancer Patients in a Tertiary Cancer Care Center. Journal of Orofacial Sciences, 11, 93-99. https://doi.org/10.4103/jofs.jofs $68 \quad 19$

[17] Oji, C. and Chukwuneke, F. (2012) Poor Oral Hygiene May Be the Sole Cause of Oral Cancer. Journal of Maxillofacial and Oral Surgery, 11, 379-383. 
https://doi.org/10.1007/s12663-012-0359-5

[18] Gaonkar, P.P., Patankar, S.R., Tripathi, N. and Sridharan, G. (2018) Oral Bacterial Flora and Oral Cancer: The Possible Link? Journal of Oral and Maxillofacial Pathology, 22, 234-238. https://doi.org/10.4103/jomfp.JOMFP $89 \quad 16$

[19] Ashreen, S., Ahmed, A., Hasan, N., Akhtar, W. and Hossain, M. (2020) Isolation and Identification of Gram-Negative Bacteria from Oral Cancer Site Infections and Study of Their Antibiotic Resistance Pattern. Bangladesh Journal of Microbiology, 36, 85-90. https://doi.org/10.3329/bjm.v36i2.45533

[20] Gaynes, R., Weinstein, R.A., Edwards, J.R. and National Nosocomial Infections Surveillance System (2005) Overview of Nosocomial Infections Caused by Gram-Negative Bacilli. Clinical Infectious Diseases, 41, 848-854. https://doi.org/10.1086/432803

[21] Horan, T.C., Garner, J.S., Jarvis, W.R., Emori, T.G. and Hughes, J.M. (1988) CDC Definitions for Nosocomial Infections, 1988. American Journal of Infection Control, 16, 128-140. https://doi.org/10.1016/0196-6553(88)90053-3

[22] Carpenter, C.B. (1990) Immunosuppression in Organ Transplantation. New Eng land Journal of Medicine, 322, 1224-1226. https://doi.org/10.1056/NEJM199004263221709

[23] Cairncross, S., Hunt, C., Boisson, S., Bostoen, K., Curtis, V., Fung, I.C.H. and Schmidt, W.-P. (2010) Water, Sanitation and Hygiene for the Prevention of Diarrhoea. International Journal of Epidemiology, 39, i193-i205. https://doi.org/10.1093/ije/dyq035

[24] Gupta, A. (2002) Hospital-Acquired Infections in the Neonatal Intensive Care Unit-Klebsiella pneumoniae. Seminars in Perinatology, 26, 340-345. https://doi.org/10.1053/sper.2002.36267

[25] Ali, A., Pineda, L.A. and Saliba, R.G. (2006) Effect of Oral Decontamination with Chlorhexidine on the Incidence of Nosocomial Pneumonia: A Meta-Analysis. Critical Care, 10, Article No. R35. https://doi.org/10.1186/cc4837

[26] Rocio, R.C., Florence, L.G., Krista, R., Gilles, R., Michèle, V., Stephanie, G., Geneviève, H., Georges, B. and Sylvie, B. (2015) Pseudomonas Aeruginosa and Periodontal Pathogens in the Oral Cavity and Lungs of Cystic Fibrosis Patients: A Case-Control Study. Journal of Clinical Microbiology, 53, 1898-1907. https://doi.org/10.1128/jcm.00368-15

[27] Minah, G.E., Solomon, E.S. and Chu, K. (1985) The Association between Dietary Sucrose Consumption and Microbial Population Shifts at Six Oral Sites in Man. Archives of Oral Biology, 30, 397-401. https://doi.org/10.1016/0003-9969(85)90066-4

[28] Dhand, A. and Snydman, D.R. (2009) Mechanism of Resistance in Metronidazole. In: Mayers, D.L., Ed., Antimicrobial Drug Resistance. Infectious Disease, Humana Press, New York, 223-227. https://doi.org/10.1007/978-1-59745-180-2 19

[29] Leiros, H.K., Kozielski-Stuhrmann, S., Kapp, U., Terradot, L., Leonard, G.A. and McSweeney, S.M. (2004) Structural Basis of 5-Nitroimidazole Antibiotic Resistance: The Crystal Structure of NimA from Deinococcus radiodurans. Journal of Biological Chemistry, 279, 55840-55849. https://doi.org/10.1074/jbc.M408044200

[30] Patel, G. and Bonomo, R.A. (2013) "Stormy Waters Ahead": Global Emergence of Carbapenemases. Frontiers in Microbiology, 4, Article No. 48. https://doi.org/10.3389/fmicb.2013.00048

[31] Bud, R. (2007) Penicillin: Triumph and Tragedy. Oxford University Press, Oxford. 